\title{
The implication of the lexicon contrastive analysis of colors in Persian \& English in perception and translation of colors
}

\author{
Hamideh Sadat Bagherzadeh \& Aqil Izadysadr*
}

\begin{abstract}
Color is all around but it is not everywhere treated in the same way. The terms people use to describe colors give another means of exploring the relationship between different languages and cultures. One field we can see the manifestation of this relationship is translation because we sometimes cannot directly translate color words from one language to another without introducing subtle changes in meaning, or the perception of the same colors in different languages may be different due to different cultures (Wardhaugh, 2006). This study aims at investigating the contrastive comparison of color lexicons- in terms of number and variety as well as their perception- in Persian and English and its effect on translation; moreover, by utilizing this cross-cultural study of color perception, want to investigate whether or not this research is in harmony with previous research -specifically the Weak Version of Linguistic Relativity Hypothesis advocating that language influences perception. The findings of this study are the result of a long process of investigating many English and Persian dictionaries, art and graphic books, personal interviews with artists, and some tasks for translators and even native speakers of both languages. After finding the specific types and lexicons of colors, we found some colors with no equivalent in the other language. Therefore, those color terms were the stimulus for our tasks. We provided some tasks and asked 50 English-Persian translators who were Persian native speakers and 50 English native speakers (Because of the limitation of access to English-Persian translators who are also English native speakers) for their perception of those colors in order to find out how perception of the colors with no equivalent in the native language would affect the translation. Results revealed that $98 \%$ of translators had difficulty perceiving and translating the two different color lexicons for which Persian had no equivalent, and 99\% of translators had difficulty translating 15 different color lexicons, which were chosen among 32 color lexicons of Persian with no English equivalent. Similarly, 97\% of English native speakers had difficulty perceiving the translated Persian color lexicons to English, for which they had no certain equivalent. Having compared the results of data collection in Persian and English, the researchers found that there are statistically significant similarities and differences between Persian and English color terms. Comparing the results revealed that the two languages are similar in the number of basic colors; however, color types are more various in Persian while there are more terms for one single color in English; therefore, the marked similarity of color grouping in Persian and English suggests some evidence that color grouping is universal and is inconsistent with the Linguistic Relativity Hypothesis. However, part of the results, which shows differences in the perception of colors in two languages, is consistent with the Weak Version of Linguistic Relativity Hypothesis.
\end{abstract}

* Authors: Hamideh Sadat Bagherzadeh, University of Wisconsin Milwaukee (UWM) (Bagherz2@uwm.edu) and Aqil Izadysadr, University of Wisconsin Milwaukee - Waukesha (UWM) (aqil@uwm.edu). 
Consequently, as an implication of the study in EFL/ESL and translation, the study provides some evidence which could be a source in translation of color terms in Persian and English.

Keywords. lexicon; contrastive analysis; colors; translation; perception; Linguistic Relativity Hypothesis (LRH)

1. Introduction. All languages have words for various colors with lexical numerical differences associated with various color names and color types. Colors seem to be highly associated with cognitive perception, culture, and, language. Species equipped with visual faculties perceive colors differently, and in humans, the richness of colors has given birth to the color lexical abundance across languages. Moreover, the use of particular colors in various cultures has made some colors more lexically abundant than others. Interestingly, each culture seems to have favored some colors over others by adding to the number of names associated with those favored colors, and Persian and English are no exception. Furthermore, this preference can be noticed in developing vocabulary for some favored color types across languages.

Moreover, Language embellishment, through the use of literature to convey abstract ideas, could have played a role in adorning the color spectrum by adding numerous words to describe a single tone or hue in various languages. The use of color words in literature has given rise to a full array of shades, hues, names, and types for colors across various languages. Throughout this research, the researchers have tried to analyze the color lexicons in Persian and English in order to investigate the differences in the number of color lexicons and the variety of color types.

The languages compared in this research are Persian and English, both of which have a rich vocabulary for color names and color types. Both languages are brimmed with loanwords from other languages, and this has certainly played a major role in giving birth to a rich vocabulary for color words in both languages. By comparing the number of color names and color types in the aforementioned languages, it can be seen that each language has specialized in amassing either more color names or color types.

These differences seem to have created an obstacle for translators to convey the true notion of a color from Persian to English and vice versa. For instance, translators usually resort to making general statements about a particular color name or type in English in order to facilitate translating that color to Persian. Consequently, the same generalization for color translation should be made while translating from Persian to English. Unfortunately, this might even make it more difficult to translate and to convey literary text with many words describing color names and types from either English to Persian or Persian to English while trying to preserve the essence of a color in a given language.

This study can help to improve the understanding of color perception in both English and Persian by facilitating the translation of color terms from English to Persian and vice versa. Moreover, while this study is inconsistent with Linguistic Relativity Hypothesis, it might provide support for the weak version of Linguistic Relativity Hypothesis via the evidence of different perceptions of color lexicons or color types with no equivalent in one's native language. Therefore, the research questions and hypotheses of this study would be as follows:

Q1: Is there any significant difference between Persian and English in regard with the number of color lexicons and the variety of color types? 
Q2: Does the comparative analysis of color lexicons in Persian and English provide any evidence in accordance with Language Relativity Hypothesis?

H1: There is no significant difference between Persian and English in regard with the number of color lexicons and the variety of color types.

H2: The comparative analysis of color lexicons in Persian and English provides no evidence in accordance with Language Relativity Hypothesis

2. Review of literature. Categorization is a fundamental property of human cognition (Roberson, 2005). In early 1970s, in a series of studies, Heider (Heider \& Olivier, 1972; Heider, 1972a, 1973, 1975) discovered that a remote type of hunter-gatherer tribe, called the Dugum Dani, seemingly manifested similar color cognitive organization as American English speakers while utilizing only two main terms for the entire visible color range (but see Heider 1972b). The results have been mainly believed to support the idea that a specific group of color categories might apply to all humans universally, which can go beyond verbal differences and be inherently specified (e.g. Rosch, 1975b; Bornstein, 1985; Soja, 1994). The categorization of color has been suggested to be an extreme case of linguistic influence on cognition (Brown \& Lenneberg, 1954; Ratner, 1989) and the opposite -an instance of the total independence of thought and language (Bornstein, 1985). Color naming accepted model suggests that there are 11 basic color terms, representing 11 common perceptual experiences. These color terms display higher processing salience due to a claimed connection between perception, visual neurophysiology, and cognition (Jameson \& Alvarado, 2003). Another study (Roberson \& Davidoff, 2000) indicates that categorical perception can be affected directly by language via the utilization of verbal labeling. In a discrimination task requiring memory demands, Roberson and Davidoff suggested that people tend to label colors with one category or another and retain these labels in their memory. They can then use them to distinguish between colors. (Özgen \& Davies, 2002).

The terms people use to describe colors give us another means of exploring the relationships between different languages and cultures (Wardhaugh, 2006, p. 235). According to Wardhaugh (2006), the way that different languages refer to colors is interesting, and sometimes, the direct translation of color words from one language to another without introducing subtle changes in meaning, e.g., English brown and French brun is difficult. There are eleven rudimentary color perceptual categories for humans, to which other basic color terms refer. Moreover, fewer terms for color are associated with simple cultures and technologies, while more complex cultures and technologies have numerous terms for color (Berlin \& Kay, 1969, p.104). All other terms for colors are combinations like grayish-brown, variations like scarlet modifications like fire-engine red, and finally the kinds of designations favored by paint and cosmetic manufacturers (Wardhaugh, 2006, p. 235).

According to Berlin and Kay (1969), an analysis of the basic color terms found in a wide variety of languages reveals certain very interesting patterns. If a language has only two color terms, they are for equivalents to black and white (or dark and light). If a third is added, it is red. The fourth and fifth terms will be yellow and green, but the order may be reversed. The sixth and seventh terms are blue and brown. Finally, as in English, come terms like gray, pink, orange, and purple, but not in any particular order. In this view there are only eleven basic color terms. 
One long-standing claim concerning the relationship between language and culture is that the structure of a language determines the way in which speakers of that language view the world. A somewhat weaker version is that the structure does not determine the world-view but is still extremely influential in predisposing speakers of a language toward adopting a particular world-view. Today, the claim is usually referred to as the Linguistic relativity hypothesis, Sapir-Whorf hypothesis, or the Whorfian hypothesis. (Wardhaugh, 2006, p. 221-222)

It is worth mentioning another study which suggests that in many languages, the term "blue" does not exist, and instead of that, people refer to short-wavelength stimuli "green" or "dark" (Lindsey \& Brown, 2002). Interestingly, in low-UV regions in the world, the word "blue" exists in languages in those regions, and in high-UV regions, there are numerous cases of languages without the word "blue." Moreover, speakers of languages without the world "blue" display blue-yellow color vision deficiency (Lindsey \& Brown, 2002). On the other hand, it might be that the meanings of color terms can refer to a particular way of life across different cultures (Phillip, 2006). There is a suggestion that natural kind terms are learned prior to the meanings of color terms during child development. There is also the claim that object terms are found more salient than property terms (Gumperz \& Levinson, 1991). In another study, it was found that color categorization can happen before language development and is independent of it (Schirillo, 2001). Color terms connotate different meanings, and they are used in proverbs of a language to transfer positive or negative meanings. Proverbs are vast in imagery, for example, food, colors, cloths, parts of body, and animals (Rashidi \& Ghaedi, 2013). The meanings of colors can also refer to positive or negative qualities; the color black has a non-euphemistic meaning among other colors, which can refer to the darkness of night, death, decay, and evil deeds. On contrary, white has been associated with light and purity, which are positive connotations. The color gray is used for dullness. The color yellow is used to refer to cowards and cheap paper, and sometimes it is used to refer to Asiatic people. On the other hand, red is connected with blood of any kind. The color green has been associated with living vegetation, and its negative connotation comes in light when it refers to the color of illness or jealousy. Blue can also be used negatively to refer to fear, fighting, and bad language and behavior. Other color terms referring to precious metals such as silver and gold are usually used positively (Allan, K., 2009).

The color domain can have semantic universals, which limit the types of basic color lexicons. These limitations come from the structure and function of the visual system. Therefore, it can be suggested that in the case of color, perception determines language rather than language determining perception (Kay \& McDaniel, 1978).

3. Methodology. This section focuses on the methodology of this research. The current study is the result of a lexicon contrastive analysis of colors in Persian \& English, which has been studied by the researchers through a long and time consuming process of searching and investigating many English and Persian dictionaries, art and graphic books, personal interviews of artists, and even ordinary native speakers of both languages.

3.1. PARTICIPANTS. Color lexicons of 11 suggested basic color terms in Persian \& English were chosen based on the color naming accepted model. There were 50 English-Persian translators and 50 native speakers of English as participants for answering the perception questions of the colors with no equivalent in the other languages. 
3.2. DATA COLLECTION AND MATERIALS. In order to collect data, i.e. the color lexicons in English and Persian, English Britannica encyclopedia and English dictionaries, including Longman, Heritage, Webster, and Oxford, Persian dictionaries, including Hayyem, Dehkhoda, Amid, and art and graphic books, including the books painting with oil paints and The Art of Color, and personal interviews with artists and even ordinary native speakers of both languages in case of special color terms have been utilized. Moreover, some part of the interviews was related to gathering data about perceiving colors with no equivalent in the native language of some English-Persian translators and English native speakers.

3.3. PROCEDURE. The aforementioned sources were investigated thoroughly in order to collect the color lexicons. Having the data at hand, the researchers used Microsoft Office Excel software in order to present the obtained data numerically and graphically in tables and comparative diagrams so as to have a better demonstration of the data. Therefore, the data were demonstrated in different tables and diagrams for both Persian and English separately as well as comparatively. Having the tables and diagrams ready, the researchers analyzed the data in a comparative contrastive method in terms of frequency of the lexicons for each of the 11 color terms and the variety of color types.

Finding the specific types and lexicons with no equivalent in one of the languages, the researchers did some interviews with 50 English-Persian translators who were Persian native speakers and 50 English native speakers (Because of the limitation of access to English-Persian translators who are also English native speakers, just ordinary English native speakers were asked for their perception of those colors.) in order to find out how perception of the colors with no equivalent in the native language would be for the native speakers and translators and how the perception would affect the translation.

\section{Results and data analysis.}

4.1. DESCRIPTIVE STATISTICS OF THE CONTRASTIVE ANALYSIS OF THE NUMBER OF COLOR LEXICONS (PERSIAN \& ENGLISH).

THE RESULTS OF THE ANALYSIS OF THE NUMBER OF THE COLOR LEXICONS FOR 11 COLORS (RED, Blue, Yellow, Green, Orange, Violet, White, Black, Gray, Brown, \& Pink) (English).

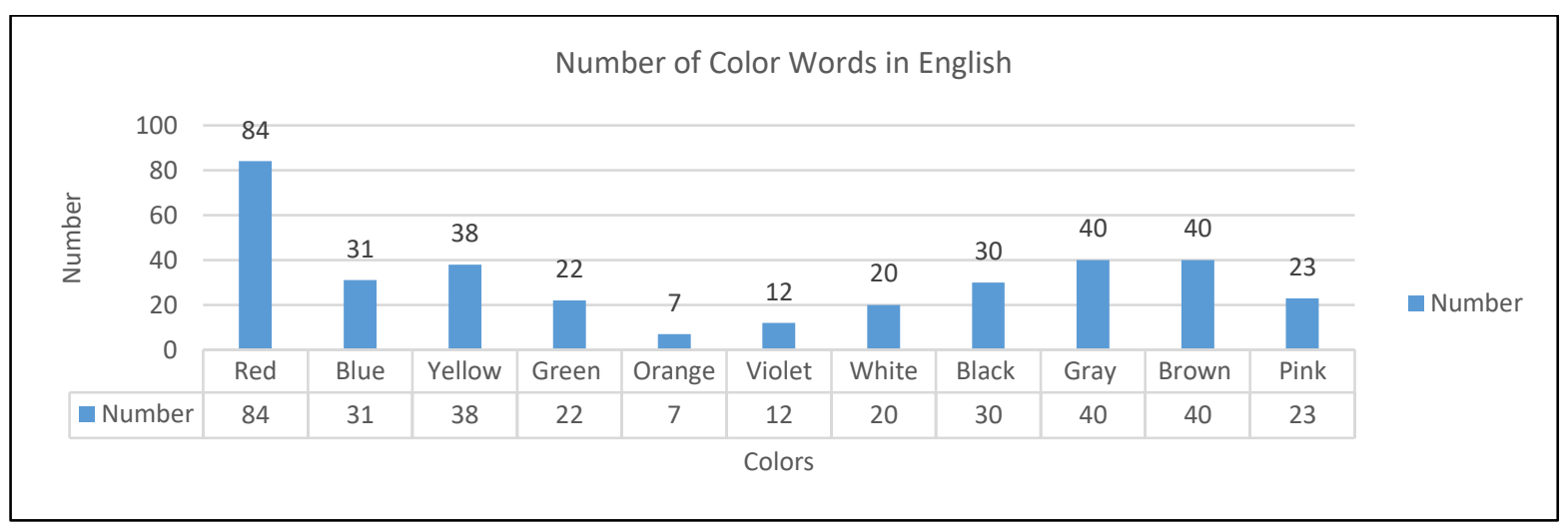

Figure 1. Descriptive Statistics of the Contrastive Analysis of the number of the Color Lexicons (English)

Comparing the number of color lexicons for every one of 11 colors in English indicates that the color Red with the total number of (84) and the color Orange with the total number of (7) 
have the most and the least number of lexicons, respectively. Following Red, the colors Gray \& Brown (40), Yellow (38), Blue (31), Black (30), Pink (23), Green (22), White (20), Violet (12), \& Orange (7) are from the highest number to the lowest, respectively.

THE RESUlTS OF THE ANALYSIS OF THE NUMBER OF THE COLOR LEXICONS FOR 11 COLORS (RED, Blue, Yellow, Green, Orange, Violet, White, Black, Gray, Brown, \& Pink) (Persian).

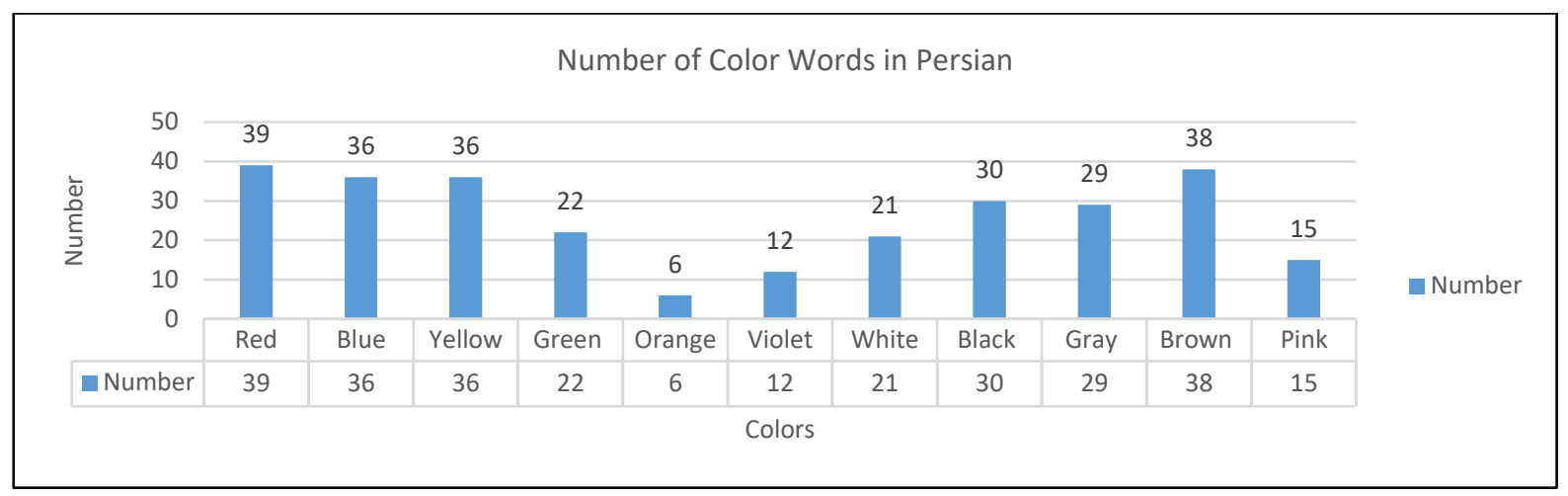

Figure 2. Descriptive Statistics of the Contrastive Analysis of the number of the Color Lexicons (Persian)

Comparing the number of color lexicons for every one of 11 colors in Persian indicates that the color Red with the total number of (39) and the color Orange with the total number of (6) have the most and the least number of lexicons, respectively. Following Red, the colors Brown (38), Blue \& Yellow (36), Black (30), Gray (29), Green (22), White (21), Pink (15), Violet (12), \& Orange (6) are the colors with the highest to the lowest number of lexicons respectively.

THE RESULTS OF THE ANALYSIS OF THE NUMBER OF THE COLOR LEXICONS FOR 11 COLORS (RED, Blue, Yellow, Green, Orange, Violet, White, Black, Gray, Brown, \& Pink) (Persian \& ENGLISH).

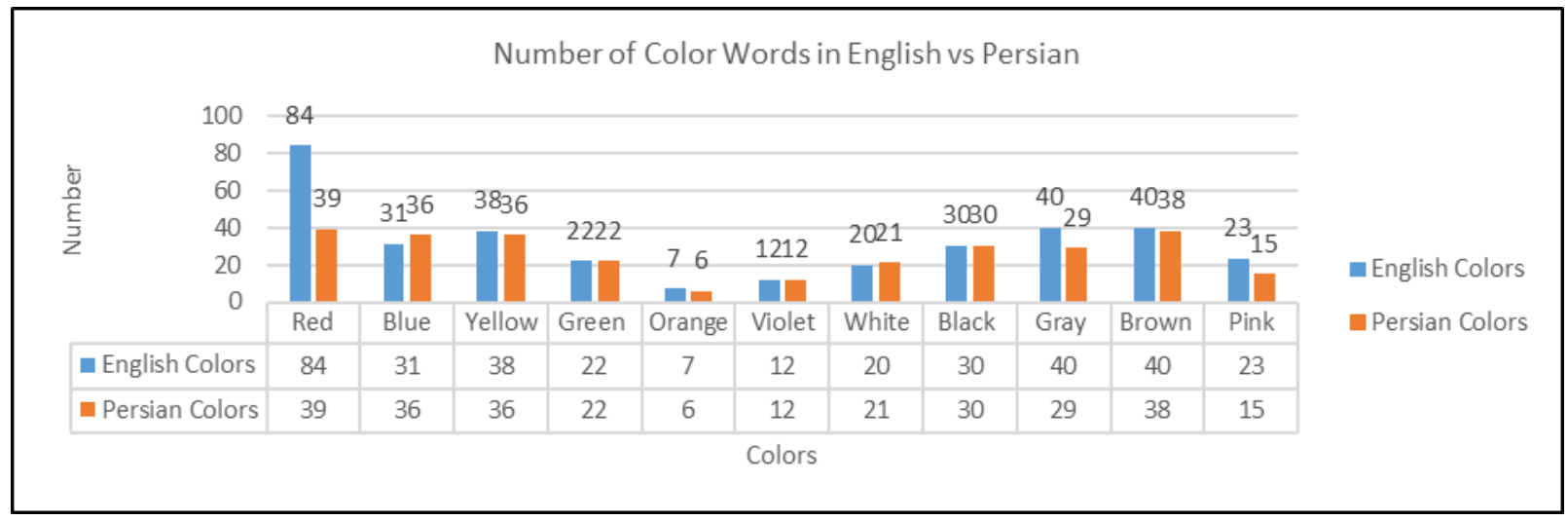

Figure 3. Descriptive Statistics of the Contrastive Analysis of the number of the Color Lexicons (English \& Persian)

Comparing the number of color lexicons for every one of 11 colors in English \& Persian indicates that the colors Red, Yellow, Orange, Gray, Brown, and Pink have more lexicons in English compared with Persian, among which Red (English (84)/ Persian (39)) has a significant 
difference in number in English and Persian. Green (22), Violet (12), and Black (30) have exactly the same number of lexicons in both English and Persian. Blue and White have fewer lexicons in English compared with Persian. Overall, Comparing the number of color lexicons in Persian and English indicates that English has more color terms for every specific color compared with Persian.

4.2. DeSCRIPTIVE STATISTICS OF THE CONTRASTIVE ANALYSIS OF THE TYPE OF COLOR LEXICONS (PERSIAN \& ENGLISH).

THE RESULTS OF THE ANALYSIS OF THE VARIETY OF THE COLOR TYPES FOR 11 COLORS (RED, BLUE, Yellow, Green, Orange, Violet, White, Black, Gray, Brown, \& Pink) (ENGlish).

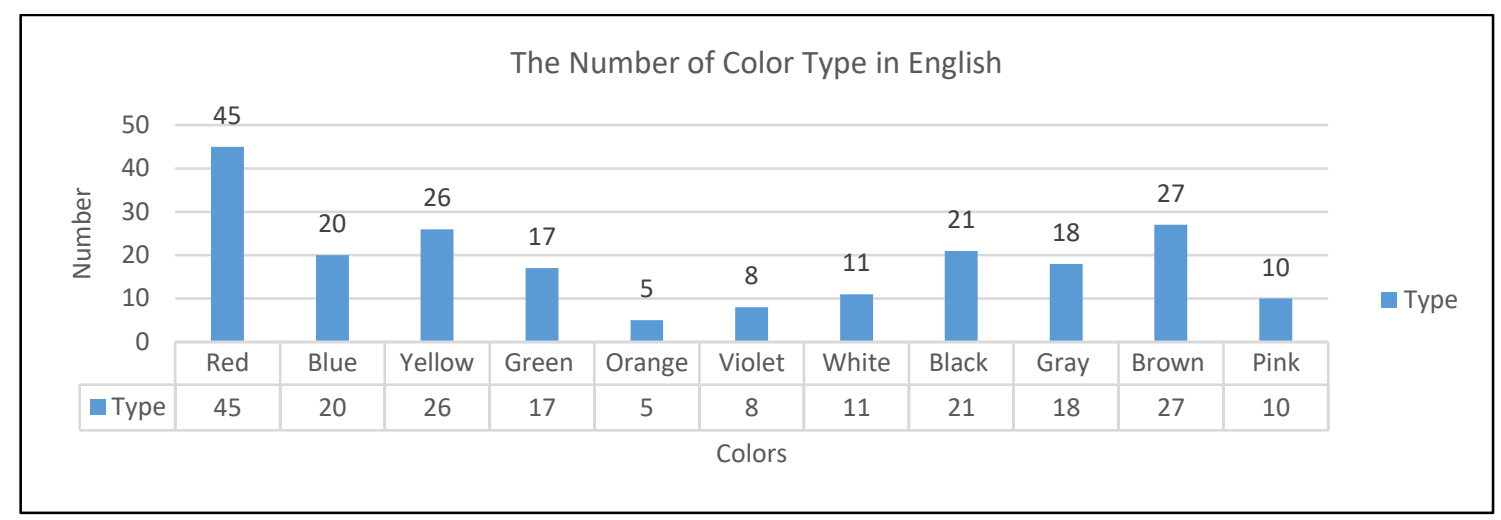

Figure 4. Descriptive Statistics of the Contrastive Analysis of the number of Color Types (English)

Comparing the number of color types for every one of 11 colors in English indicates that the color Red with the total number of (45) and the color Orange with the total number of (5) have the most and the least number of types, respectively. This shows that regarding both the number of color lexicons and the number of color types, the color red has the highest number and the color of orange has the lowest number. After Red (45), the colors Brown (27), Yellow (26), Black (21), Blue (20), Gray (18), Green (17), White (11), Pink (10), Violet (8), \& Orange (5) are the colors with the highest to the lowest number of types, respectively. These colors are almost in the same order as the color lexicons except for subtle changes in the order of some colors. 
THE RESUlTS OF THE ANALYSIS OF THE VARIETY OF THE COLOR TYPES FOR 11 COLORS (RED, Blue, Yellow, Green, Orange, Violet, White, Black, Gray, Brown, \& Pink) (Persian).

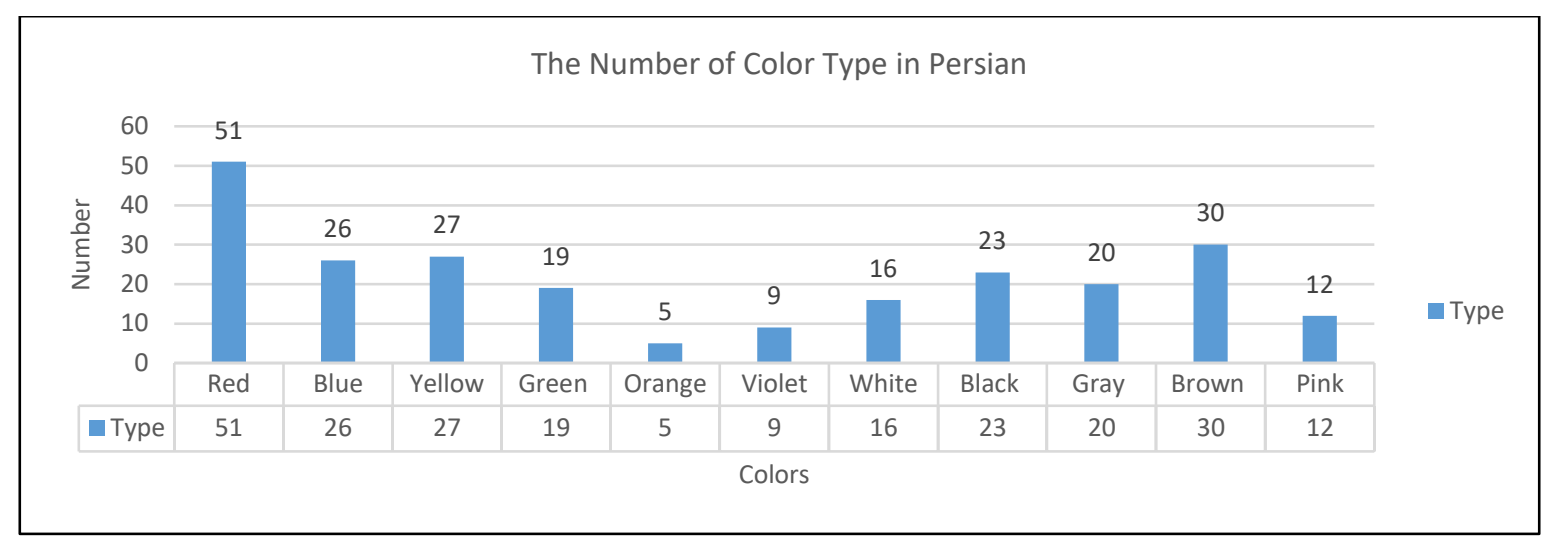

Figure 5. Descriptive Statistics of the Contrastive Analysis of the number of Color Types (Persian)

Comparing the number of color types for every one of 11 colors in Persian indicates that the color Red, with the total number of (51), and the color Orange, with the total number of (5), have the most and the least number of types, respectively. In other words, regarding both the number of color lexicons and the number of color types, the color red has the highest number and the color of orange has the lowest number. Following Red (51), the colors Brown (30), Yellow (27), Blue (26), Black (23), Gray (20), Green (19), White (16), Pink (12), Violet (9), \& Orange (5) are the colors with the highest to the lowest number of types, respectively. These colors are almost in the same order as the color lexicons except for subtle changes in the order of some colors.

THE RESULTS OF THE ANALYSIS OF THE VARIETY OF THE COLOR TYPES FOR 11 COLORS (RED, BLUE, Yellow, Green, Orange, Violet, White, Black, Gray, Brown, \& Pink) (English \& PERSIAN).

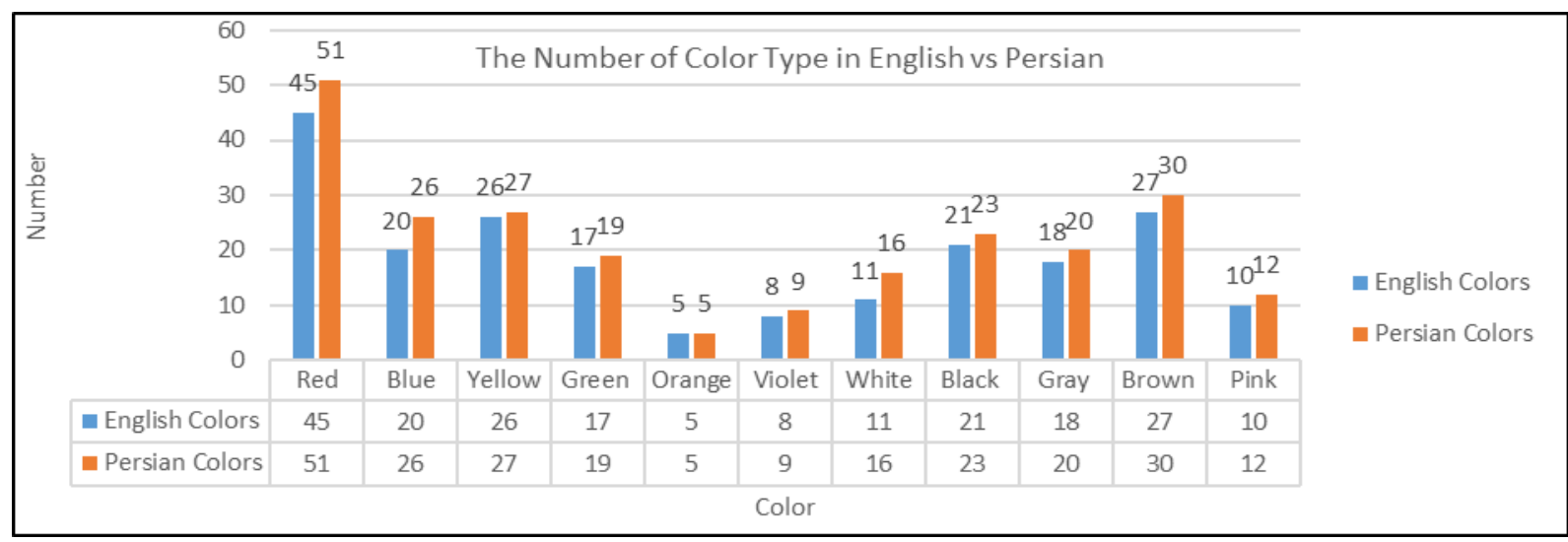

Figure 6. Descriptive Statistics of the Contrastive Analysis of the number of Color Types (Persian \& English)

Overall, Comparing the variety of color types in Persian and English indicates that Persian has more color types for every specific color compared with English. This difference is the highest for the color Red (English (45 types), Persian (51types)); nonetheless, the difference for 
the other colors is not very significant, and the number of color types for the color Orange (5 types) is the same in both English and Persian. The order of the colors from the highest number of types to the lowest is almost the same in Persian and English except for the switch of the colors Black (21) and Blue (20) in English to Blue (26) and Black (23) in Persian. In addition, the results of the interviews with English-Persian translators and English native speakers revealed that $98 \%$ of translators had difficulty perceiving and translating the two different color lexicons for which Persian had no equivalent, and 99\% of translators had difficulty translating 15 different color lexicons, which were chosen among 32 color lexicons of Persian with no English equivalent. Similarly, $97 \%$ of English native speakers had difficulty perceiving the translated Persian color lexicons to English, for which they had no certain equivalent.

5. Discussion. According to the findings, considering the first question of this study (Q1: Is there any significant difference between Persian and English in regard with the number of color lexicons and variety of color types?), the analysis of the obtained data revealed that regarding the number of color lexicons, English is more resourceful compared with Persian; however, considering the types (subcategories) of colors, Persian is richer and has more varieties of colors compared with English. As a result, the first null hypothesis (H1: There is no significant difference between Persian and English in regard with the number of color lexicons and variety of color types.) is rejected; nonetheless, it is worth mentioning that in both cases, except for the color Red, the differences are not significant. regarding the fact that the significant difference is just for a few cases, and since few observed cases of significant differences may not be counted as statistically significant, one may argue that it still remains null. No matter how significant the difference was, what matters for this study is how comparatively different the two languages are.

Considering the second question (Q2: Does the comparative analysis of color lexicons in Persian and English provide any evidence in accordance with Language Relativity Hypothesis?), the analysis of obtained data revealed that English and Persian have similar color grouping systems with 11 basic color categories. Moreover, the number of color lexicons and the variety of color types (subcategories)- except for few cases and subtle differences- are very similar. Therefore, the marked similarity of color grouping in Persian and English suggests other evidence that color grouping is universal and is inconsistent with the Linguistic Relativity Hypothesis. As a result, the second null hypothesis (H2: The comparative analysis of color lexicons in Persian and English provides no evidence in accordance with Language Relativity Hypothesis) is also rejected.

Regarding the LRH, it is noteworthy to mention that there were some differences in perception of colors in two languages, which is consistent with the Weak Version of Linguistic Relativity Hypothesis. Asking artists and translators for their perception of the colors with no equivalent in their language, it seemed that since there was not an equivalent for those color lexicons or types, it was difficult for them to perceive those colors vividly and to translate them to their language easily and certainly. For example, the color Imperial green in English was difficult to be perceived and understood by Persian speakers, and the color (Ghermez-e Gol Anari, i.e. red of the same color as pomegranate flowers) was difficult to be perceived and understood by American speakers. Furthermore, the researchers are willing to mention that the findings are based on the achieved data, and while they tried hard to collect the data thoroughly, there may be color lexicons or types which have been missed due to the limitations of the study. 
6. Conclusion. The purpose of this study was to compare English and Persian color lexicons contrastively in terms of the number of color lexicons and the variety of color types in both languages in order to investigate how they differ, and whether or not the differences are significant. In addition, the researchers sought to find the possible evidence regarding the consistency or inconsistency of the findings with Linguistic Relativity Hypothesis.

The findings of the data analysis revealed that indeed there are differences in the number of color lexicons and color types in Persian and English; however, except for the color Red, the differences are not conspicuous to the extent to indicate a different system and different world views. Additionally, the similar system of 11 colors in Persian and English and the similarity of color grouping in Persian and English suggests another evidence that color grouping is universal and is inconsistent with the Linguistic Relativity Hypothesis. Nevertheless, in case of counter arguments and further studies, a few cases of differences in color grouping in Persian and English, which affected the perception of colors and the translation of colors in these languages, might be considered consistent with the weak version of Linguistic Relativity Hypothesis.

Therefore, the outcome of the present study inspires additional research on the weak version of Linguistic Relativity Hypothesis in order to come up with a more certain conclusion in that case. Further research is suggested regarding the implication of this study on perceiving colors with no equivalent in the other languages, and how it affects the color translation and color learning in SLA. Furthermore, investigating the possible psychological or cultural reasons behind differences in some specific color lexicon number and types in Persian and English would be another intriguing study suggestion in the area of psycholinguistics related to this research. Interestingly, one other implication of the data collected via this study would be utilizing the data of equivalent tables of color lexicons of Persian and English as a mini-bilingual dictionary of color lexicons as a practical source for translators, EFL teachers and learners, and artists.

\section{References}

Allan, Keith. 2009. The connotations of English colour terms: Colour-based x-phemisms. Journal of Pragmatics 41(3).. 626-637. https://doi.org/10.1016/j.pragma.2008.06.004.

Bornstein, Marc H. 1985. On the development of color naming in young children: Data and theory. Brain and Language 26(1).. 72-93. https://doi.org/10.1016/0093-934X(85).90029-X.

Brown, Roger W. \& Eric H. Lenneberg. 1954. A study in language and cognition. The Journal of Abnormal and Social Psychology 49(3).. 454-462. https://doi.org/10.1037/h0057814.

Gumperz, John J. \& Stephen C. Levinson. 1991. Rethinking linguistic relativity. Current Anthropology 32(5). 613-623. https://doi.org/10.1086/204009.

Heider, Eleanor R. 1972a. Probabilities, sampling and ethnographic method: The case of Dani colour names. Man 7(3). 448. https://doi.org/10.2307/2800917.

Heider, Eleanor R. 1972b. Universals in color naming and memory. Journal of Experimental Psychology 93(1). 10-20. https://doi.org/10.1037/h0032606.

Heider, Eleanor R. \& Donald C. Olivier. 1972. The structure of the color space in naming and memory for two languages. Cognitive Psychology 3(2). 337-354. https://doi.org/10.1016/00100285(72).90011-4.

Itten, Johannes. 1974. The art of color: The subjective experience and objective rationale of color. Hoboken, NJ: Wiley.

Jameson, Kimberly A. \& Nancy Alvarado. 2003. Differences in color naming and color salience in Vietnamese and English. Color Research \& Application 28(2). 113-138.

https://doi.org/10.1002/col.10131. 
Kay, Paul. 1969. Basic color terms: Their universality and evolution. Berkeley: University of California Press.

Kay, Paul \& Chad K. McDaniel. 1978. The linguistic significance of the meanings of basic color terms. Language 54(3). 610-646. https://doi.org/10.1353/lan.1978.0035.

Lindsey, Delwin T. \& Angela M. Brown. 2002. Color naming and the phototoxic effects of sunlight on the eye. Psychological Science 13(6). 506-512. https://doi.org/10.1111/14679280.00489.

Philip, Gill. 2006. Connotative meaning in English and Italian colour-word metaphors. Metaphorik 10. 59-93. https://doi.org/10.6092/unibo/amsacta/2265.

Rashidi, Nasser \& Hadis Ghaedi. 2013. Contrastive discourse analysis of Persian and English animal proverbs. Babel Revue Internationale De La Taduction / International Journal of Translation 59(1). 1-24. https://doi.org/10.1075/babel.59.1.01ras.

Ratner, Carl. 1989. A sociohistorical critique of naturalistic theories of color perception. The Journal of Mind and Behavior 10(3). 361-372.

Roberson, Debi \& Jules Davidoff. 2000. The categorical perception of colors and facial expressions: the effect of verbal interference. Memory \& Cognition 28(6). 977-986. https://doi.org/10.3758/BF03209345.

Rosch, Eleanor. 1975. The nature of mental codes for color categories. Journal of Experimental Psychology: Human Perception and Performance 1(4). 303-322. https://doi.org/10.1037/0096-1523.1.4.303.

Rosch, Eleanor. 1975. Universals and cultural specifics in human categorization. In Richard W. Brislin, S. Bochner \& Walter Joseph Lonner (eds.), Cross-cultural Perspectives on Learning. 177-206. Sydney: Halstead Press.

Rosch, Eleanor. 1973. Natural categories. Cognitive Psychology 4(3). 328-350. https://doi.org/10.1016/0010-0285(73).90017-0.

Schirillo, James A. 2001. Tutorial on the importance of color in language and culture. Color Research \& Application 26(3). 179-192. https://doi.org/10.1002/col.1016.

Soja, Nancy N. 1994. Young children's concept of color and its relation to the acquisition of color words. Child Development 65(3). 918. https://doi.org/10.2307/1131428.

Wardhaugh, Ronald. 2005. An introduction to sociolinguistics. Hoboken, NJ: Wiley-Blackwell. Özgen, Emre \& Ian R. Davies. 2002. Acquisition of categorical color perception: A perceptual learning approach to the linguistic relativity hypothesis. 131(4). 477-493. Journal of Experimental Psychology: General https://doi.org/10.1037/0096-3445.131.4.477. 